\title{
High resolution drift tube hodoscopes for cosmic ray studies
}

\author{
A.D. Tomasch ${ }^{\text {a }}$ for the PBAR *, SMILI ** and HEAT *** Collaborations \\ ${ }^{a}$ Randall Laboratory, Department of Physics, University of Michigan, Ann Arbor, MI 48109-1120, USA
}

\begin{abstract}
Thin-walled drift tubes have been used in conjunction with a superconducting magnet for the rigidity spectrometer aboard two recent particle astrophysics experiments flown on high altitude balloons: PBAR (a low energy antiproton search) and SMILI (the superconducting magnet instrument for light isotopes). The HEAT (high energy antimatter telescope) experiment currently under construction will also employ this technology. This paper reviews the design, construction, and in-flight operation of the PBAR and SMILI systems, as well as the design of the HEAT system which will be used in conjunction with a new superconducting magnet aboard an upcoming series of balloon experiments to study high energy positrons and antiprotons in the cosmic radiation. In addition to a brief account of the scientific goals for these flights, the prospects for future application of this technology to long duration exposures aboard antarctic balloon flights and spacecraft are discussed.
\end{abstract}

\section{Introduction}

Much recent progress has been made toward answering a number of outstanding questions in the context of particle astrophysics by means of direct measurements of the cosmic radiation above the earth's atmosphere. These experiments have employed magnet spectrometers lofted aboard balloons to measure both particle mass and energy via the rigidity-velocity technique. Central to all these experiments is the measurement of rigidity ( $\equiv$ momentum/charge), which is accomplished by tracking particles within the field produced by a superconducting magnet. All of these experiments employ arrays of drift tubes as the tracking hodoscope. Drift tubes have proven to be ideal for use aboard balloons because of their inherent ruggedness, simplicity, low cross-talk, good performance in magnetic fields, and low mass. The latter is very important, since low background searches for species such as antiprotons hinge upon the redundant measurement of many points along an individual trajectory, which permits the rare large-angle scatterings which comprise the major background to be efficiently rejected by goodness-of-fit criteria.

\section{The PBAR spectrometer}

The PBAR experiment [1-3], shown schematically in fig. 1, was used to set a new limit [2] of $\bar{p} / p<2.0 \times$

\footnotetext{
* See ref. [1].

$* *$ See ref. [6].

$* * *$ See ref. [10].
}

$10^{-5}$ ( $85 \% \mathrm{CL}$ ) for energies below $1580 \mathrm{MeV}$. This showed the previously reported [4] antiproton signal to be in error, and demonstrated that the abundance of antiprotons at these energies is consistent with that expected from standard models for the propagation of cosmic rays in the galaxy. An elevated antiproton ratio at these energies could otherwise have been taken as direct evidence for the annihilation of relic particles within a massive galactic halo.

PBAR employed a rigidity spectrometer consisting of 323 thin-walled drift tubes close-packed within the

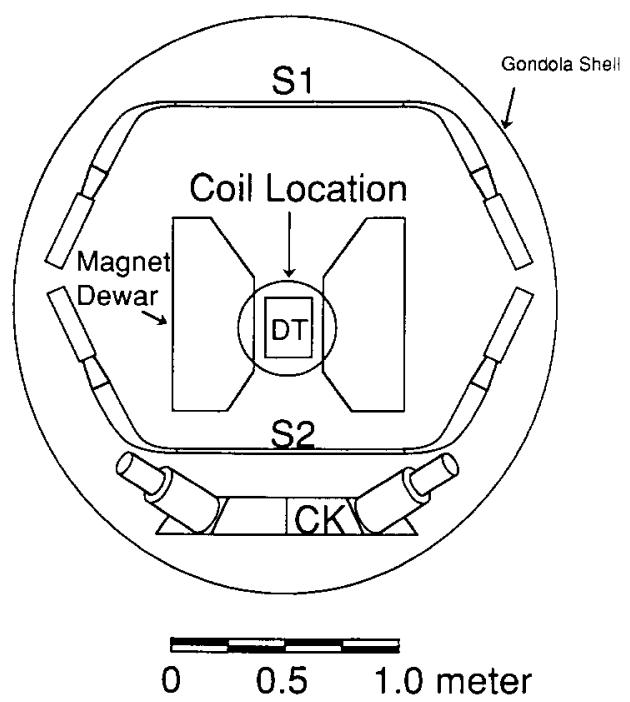

Fig. 1. Schematic cross-section of the PBAR magnetic spectrometer showing time-of-flight scintillation counters (S1, S2), threshold water Cherenkov counter (CK), magnet, and drift tube hodoscope (DT). 
room temperature bore of a superconducting magnet. The in-flight magnetic field was $\sim 9 \mathrm{kG}$, providing typical field integrals through the spectrometer of $\sim 50$ MV. The tubes were arranged in 24 layers of which 16 were parallel to the magnetic field and used for the rigidity measurement. The remaining tubes were normal to the magnetic field and used to reconstruct the particle trajectories in three dimensions. While the rigidity determination is sufficiently accurate without this additional information, the three-dimensional pointing can be used to apply mapping and angular corrections to the other counters.

The individual tubes were constructed from thinwalled plastic tubing of $1.27 \mathrm{~cm}$ diameter produced by spiral winding two layers on a mandril with contact cement between the layers. The inner layer consisted of an aluminized polycarbonate plastic $13 \mu \mathrm{m}$ thick, while the outer layer was $13 \mu \mathrm{m}$ thick mylar. This has the advantage that inner plastic layer is itself conductive, producing a cathode that is less affected by scratches in the aluminum. The final wall thickness was $\sim 30 \mu \mathrm{m}$. A vertically incident particle traversed $\sim 0.3$ g $\mathrm{cm}^{-2}(\sim 0.6 \%$ of a radiation length) of material

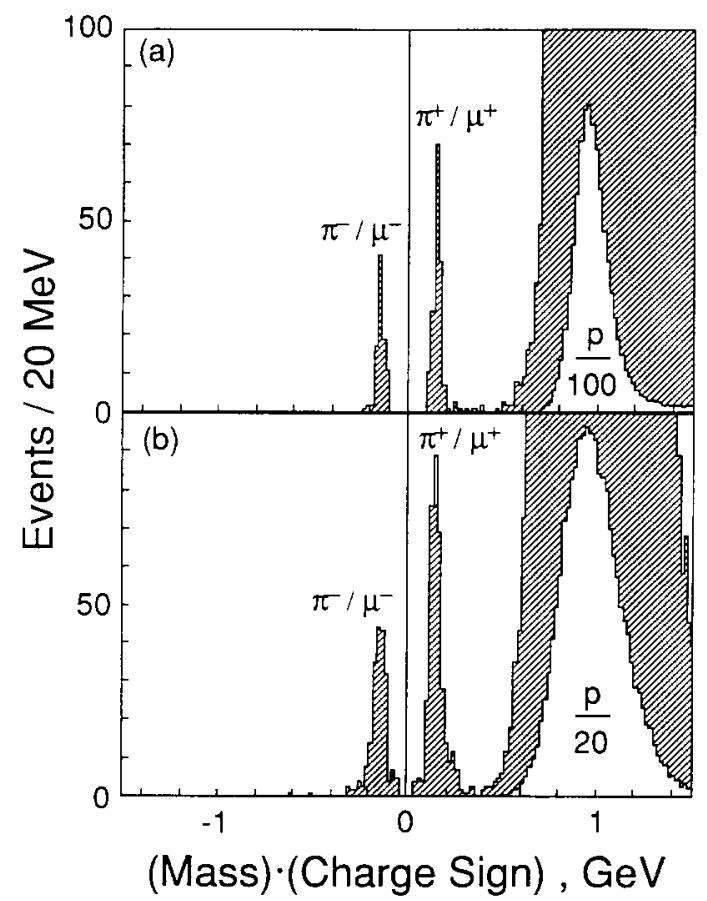

Fig. 2. Mass histograms for singly charged cosmic rays collected during the balloon flight of August 1987 (a) $0.17<\beta<$ 0.8 (LV) and (b) $0.8<\beta<0.93$ (HV). The LV data use velocities determined solely from time-of-flight measurements, while the HV data include velocity information from the water Cherenkov counter. No antiproton candidates were found, yielding a limit of $\bar{p} / p<2.0 \times 10^{-5}(85 \% \mathrm{CL})$ for energies of $100-1580 \mathrm{MeV}$. passing through the rigidity analyzing portion of the spectrometer. The array was housed in a precisionmachined aluminum box, with thin windows covering the top and bottom of the assembly to protect the tubes. Precision-machined brass plugs were used to mount the tubes within the plates, with machined delrin pins and precision-drawn tubular ferrules used to position the $20 \mu \mathrm{m}$ diameter gold-plated tungsten wires within the tubes. The wire end positions within the array were known a priori to $\sim 30 \mu \mathrm{m}$ as verified by preflight tracking tests with atmospheric muons. The tubes were operated in limited streamer mode [5] with a gas mixture of argon : ethane $1: 1$. Typical operating voltage was $2200 \mathrm{~V}$. Timing data were digitized by a LeCroy 4290 TDC system operated in common start mode, and telemetered to the ground along with pulse height data from the other counters. Single tube resolution during flight was $110 \mu \mathrm{m}$ for singly charged cosmic rays, averaged over all tubes, incident angles, and energies. A complete description of the PBAR experiment has been presented [3].

Fig. 2 illustrates the mass resolution obtained when the rigidity measurement was combined with velocity information (a) from the time-of-flight (TOF) scintillators (S1, S2) and (b) from the combination of TOF and Cherenkov (CK) information for the PBAR flight data. Average timing resolution was 160 ps for $\beta<0.8$. The resulting mass resolution for protons was $\sigma_{\mathrm{m}}=0.08$ amu.

Another critical consideration for the antiproton search was not simply to identify particles by measuring their mass, but to reject the background due to misidentified curvature for events which underwent hard nuclear scatters within the hodoscope, As illustrated in fig. 3. First, a "goodness-of-fit" parameter $\left(\chi_{R}\right)$ was defined [3]. With 16 independent position measurements along the particle trajectory, the exclusion of events populating the tail of the $\chi_{R}$ distribution efficiency rejected hard scatterings, while removing only $\sim 10 \%$ of the data sample. Fig. 3 illustrates the effect of removing eight of the drift tube layers from the analysis, with no other changes. A severe negative rigidity background is introduced, illustrating the need for many independent trajectory measurements while introducing a minimum of matter in the tracking hodoscope.

\section{SMILI: the superconducting magnet instrument for light isotopes}

SMILI [6-9] is a reconfigured version of PBAR optimized for the study of light isotopes. Data from the first flight in $1989[7,8]$ has confirmed [9] the expected rise of the isotopic ratio ${ }^{3} \mathrm{He} /{ }^{4} \mathrm{He}$ for energies of $1-3$ $\mathrm{GeV} / \mathrm{n}$, providing further evidence that light cosmic 
rays propagate in a galactic environment similar to that encountered by the medium cosmic rays $\mathrm{C}, \mathrm{N}$, and $\mathrm{O}$.

The SMILI instrument is shown in fig. 4. While formally similar to PBAR, SMILI has been optimized for larger aperture, larger field integral, and longer TOF base line. The drift tube tracking array consists of four modules each containing four layers of tubes. Within each module, the four layers are grouped into two "X" and two "Y" layers. Thus, of the 16 total layers, 8 are used for rigidity analysis. The system contains a total of 512 tubes $2.5 \mathrm{~cm}$ in diameter. The tubing is similar to that of the PBAR array, except that an additional layer of $13 \mu \mathrm{m}$ thick mylar has been added, bringing the nominal wall thickness to $\sim 40$ $\mu \mathrm{m}$. The tube and wire mount mechanics are also based on the PBAR design. The gas mixture is again argon:ethane $1: 1$, but the tubes are operated in a saturated proportional mode to reduce sensitivity to knockon electrons produced by the passage of cosmic ray ions through the instrument. Data acquisition is again by means of a LeCroy 4290 system, operated in common stop mode. To lock out late starts arriving at the 4290 system from the drift tubes after a valid trigger is received, special pulse stretching circuits have been added to the electronics. Chamber position within the instrument is established by means of a precision survey of optical targets mounted to the chambers at precisely known positions. Typical in-flight tracking resolution for a single tube is $<200 \mu \mathrm{m}$.

Fig. 5 illustrates the excellent isotopic resolution achieved for ${ }^{4} \mathrm{He}$ and ${ }^{3} \mathrm{He}$ during the balloon flight of August 31-September 1, 1989. The data for 100-300 $\mathrm{MeV} / \mathrm{n}$ employ only TOF data for velocity determination, while the $500-700 \mathrm{MeV} / \mathrm{n}$ data include information from the water Cherenkov counter. The widths of the ${ }^{4} \mathrm{He}$ peaks correspond to mass resolutions of $\sigma_{\mathrm{m}}=$ $0.10 \mathrm{amu}$ and $\sigma_{\mathrm{m}}=0.13 \mathrm{amu}$, respectively. Preliminary results for this data set have been reported [7-9]. The instrument has flown successfully a second time during the summer of 1991.

\section{HEAT: the high energy antimatter telescope}

HEAT $[10,11]$ is currently under construction. The first HEAT instrument, HEAT- $\mathrm{e}^{+} \mathrm{e}^{--}$, will measure the positron fraction $\mathrm{e}^{+} /\left(\mathrm{e}^{+}+\mathrm{e}^{-}\right)$to at least $50 \mathrm{GeV}$ to determine if the unexplained rise [12] in this ratio at

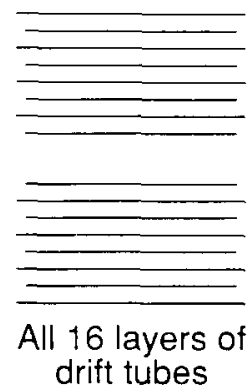

drift tubes

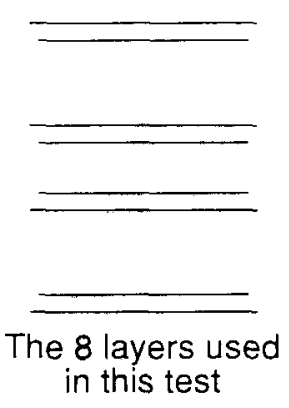

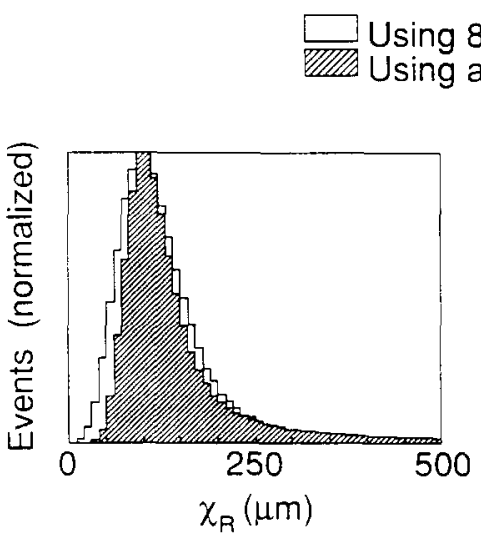

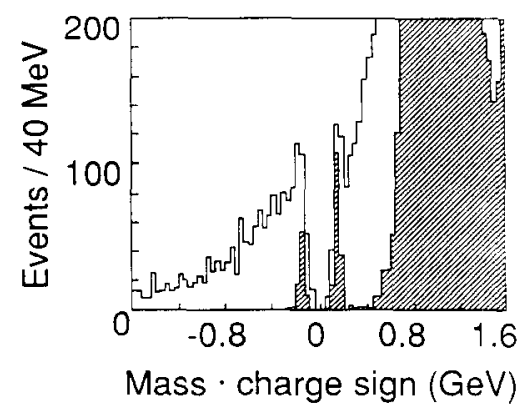

Fig. 3. The effect or removing 8 of the 16 drift tube layers from the analysis of the PBAR flight data. Even though the single-tube resolution remains at $110 \mu \mathrm{m}$, severe background in the negative rigidity plane is introduced due to large angle scatterings. 
$\sim 20 \mathrm{GeV}$ continues at higher energies. Future HEAT flights will measure the antiproton abundance at energies up to $20 \mathrm{GeV}$, by combining the rigidity spectrometer with liquid and gas based ring imaging Cherenkov (RICH) [13,14] counters. Presently the discrepancy between measured and predicted antiproton abundances at $10 \mathrm{GeV}$ is a factor of $\sim 3$ overabundance, which could indicate a source of antiprotons in addition to those expected to be produced as secondaries in cosmic ray interactions.

The HEAT- $\mathrm{e}^{+} \mathrm{e}^{-}$spectrometer is shown in fig. 6 . The rigidity spectrometer is based on a new superconducting magnet which will produce an approximately uniform field of $1 \mathrm{~T}$ within room temperature bore measuring $50 \times 51 \times 61 \mathrm{~cm}$. The magnet has been successfully tested at full field, while the cryostat is under

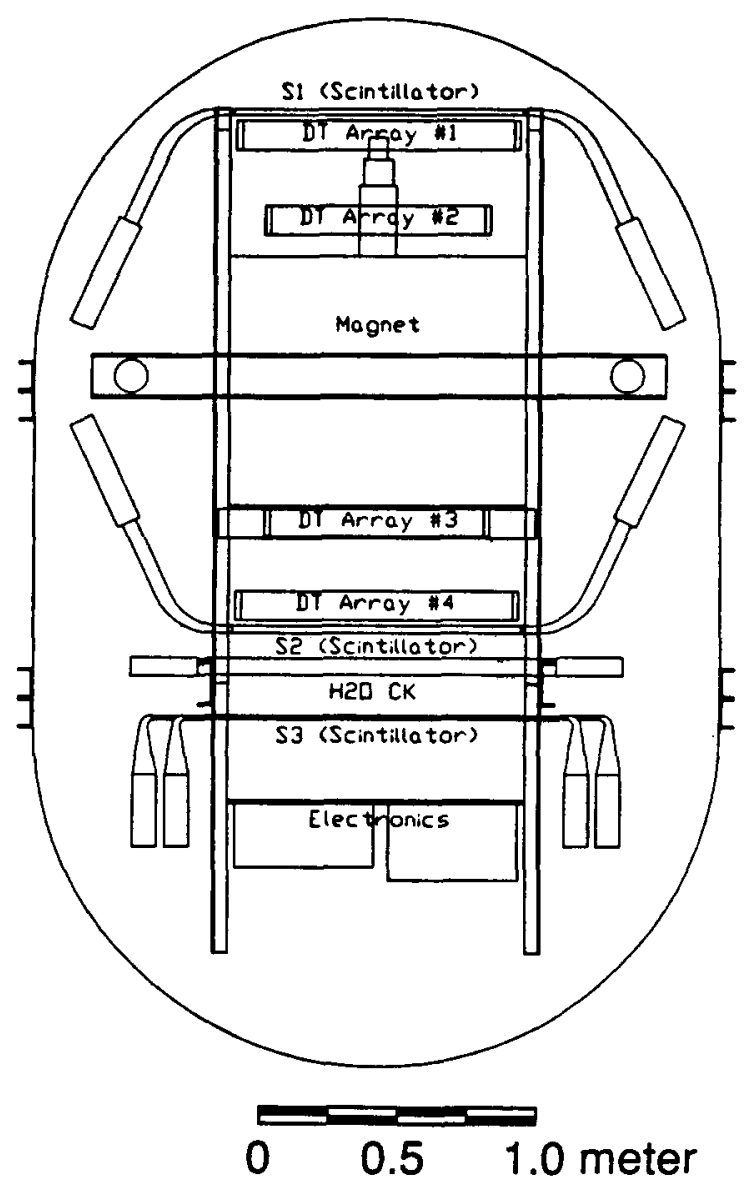

Fig. 4. The SMILI payload. While formally similar to PBAR, it has been optimized for large aperture and high resolution. The major elements are time-of-flight scintillators (S1, S2), drift tube tracking arrays (DT arrays 1-4) and water Cherenkov counter $\left(\mathrm{H}_{2} \mathrm{O} \mathrm{CK}\right)$. An additional thin scintillator (S3) is used to reject events for which the incident nucleus interacts in the Cherenkov counter. construction. The drift tube hodoscope is similar in concept to the PBAR array and consists of 479 tubes $2.5 \mathrm{~cm}$ in diameter arrayed in 26 layers. Of these, 18 are parallel to the magnetic field and used for the rigidity measurement. This spectrometer has $\sim 14$ times the aperture and $\sim 6$ times the $B \cdot l^{2}$ when compared to the PBAR instrument operated at its maximum field of $1.5 \mathrm{~T}$. Typical field integrals through the rigidity analyzing portion of the hodoscope are 150 MV. The tubing will be of the same three-layer construction used for SMILI. The mechanical design is again similar to PBAR, but the support plates and tube mechanics have been reduced to the minimum thickness practical in order to minimize the amount of aperture lost around the hodoscope perimeter due to the obstruction of the support mechanics. Transition radiation detectors (TRD) [15] and an electromagnetic shower calorimeter (SC) reject protons to 1 part in $10^{4}$, reducing the proton contamination of the positron signal to no more than $10 \%$. Up-down discrimination is accomplished by means of a time of flight (TOF) system, with the top layer of the shower calorimeter serving as the bottom TOF counter.

Several new features have been incorporated into the HEAT design to improve the single-tube resolution. Most important is the use of a low-diffusion drift gas based on $\mathrm{CO}_{2}$-isobutane. For $2.5 \mathrm{~cm}$ diameter tubes the diffusion limit to resolution is $\sim 30 \mu \mathrm{m}$. Additional steps have been taken to ensure that the wire positions are accurately known. Injection molded pins will be used to position the wires. These, in combination with a precision survey of the assembled hodoscope prior to stringing the wires, should provide wire placement accuracy in the 10-15 $\mu \mathrm{m}$ range. Computer simulations have been done which indicate that the slow drift speed inherent in $\mathrm{CO}_{2}$ based gases, in combination with the quasi-uniform magnetic field produced by the two-coil magnet, permit corrections for the $\boldsymbol{w} \times \boldsymbol{B}$ effects introduced into the electron drift to be applied, where $w$ is the mean electron drift velocity.

In order to correct for the variation of electron drift speed with temperature, pressure, and gas composition, we have developed a novel calibration cell. A single drift tube is illuminated by five collimated 10 $\mu \mathrm{Ci}{ }^{90} \mathrm{Sr}$ sources, each at a fixed radius from the sense wire. A timing spectrum from the cell exhibits five well resolved peaks. The mean of each peak corresponds to the drift time from a known fixed radius. Thus, the cell provides a direct measure of the time-to-distance relation within the tube. The timing spectrum will be read out every $5-10$ min during flight, a time scale which is short compared to typical temperature and pressure changes within the balloon gondola. Tests are currently underway with a prototype calibrator and drift tube array to measure the tracking resolution which can be achieved with this system. 


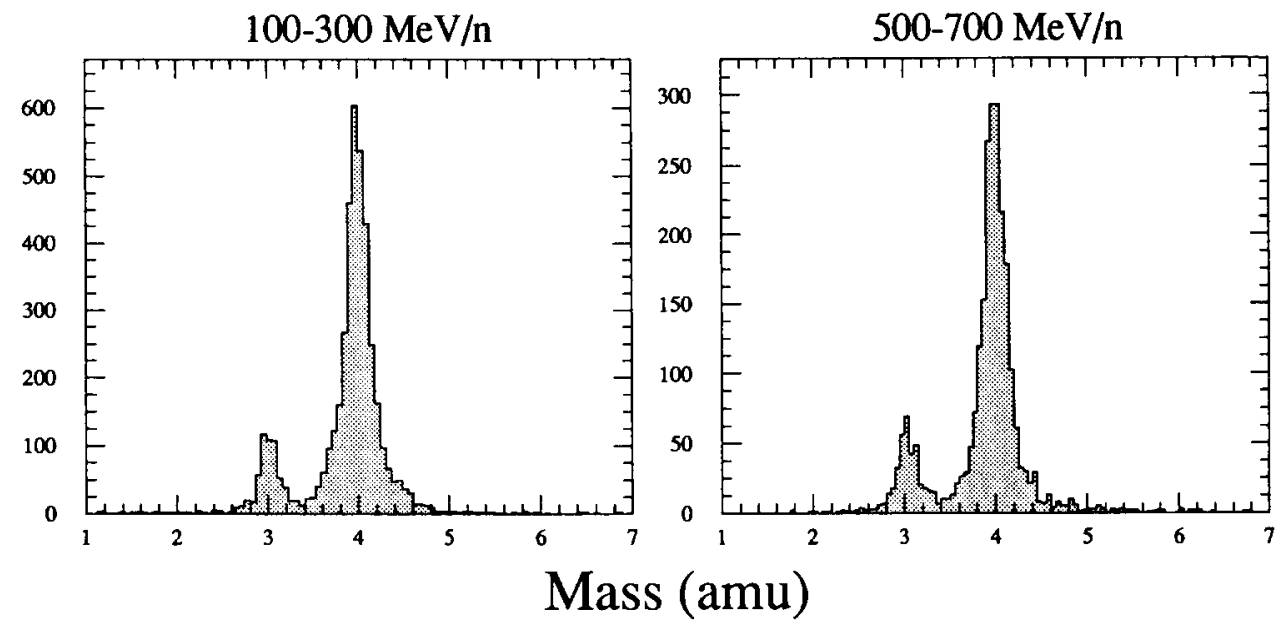

Fig. 5. Mass histograms obtained from SMILI flight data for the $100-300$ and $500-700 \mathrm{MeV} / \mathrm{n}$ energy intervals. Excellent separation of ${ }^{3} \mathrm{He}$ from ${ }^{4} \mathrm{He}$ is evident. The width of the ${ }^{4} \mathrm{He}$ peak corresponds to a mass resolution of 0.10 amu for the $100-300$ $\mathrm{MeV} / \mathrm{n}$ data and $0.13 \mathrm{amu}$ for the $500-700 \mathrm{MeV} / \mathrm{n}$ data.

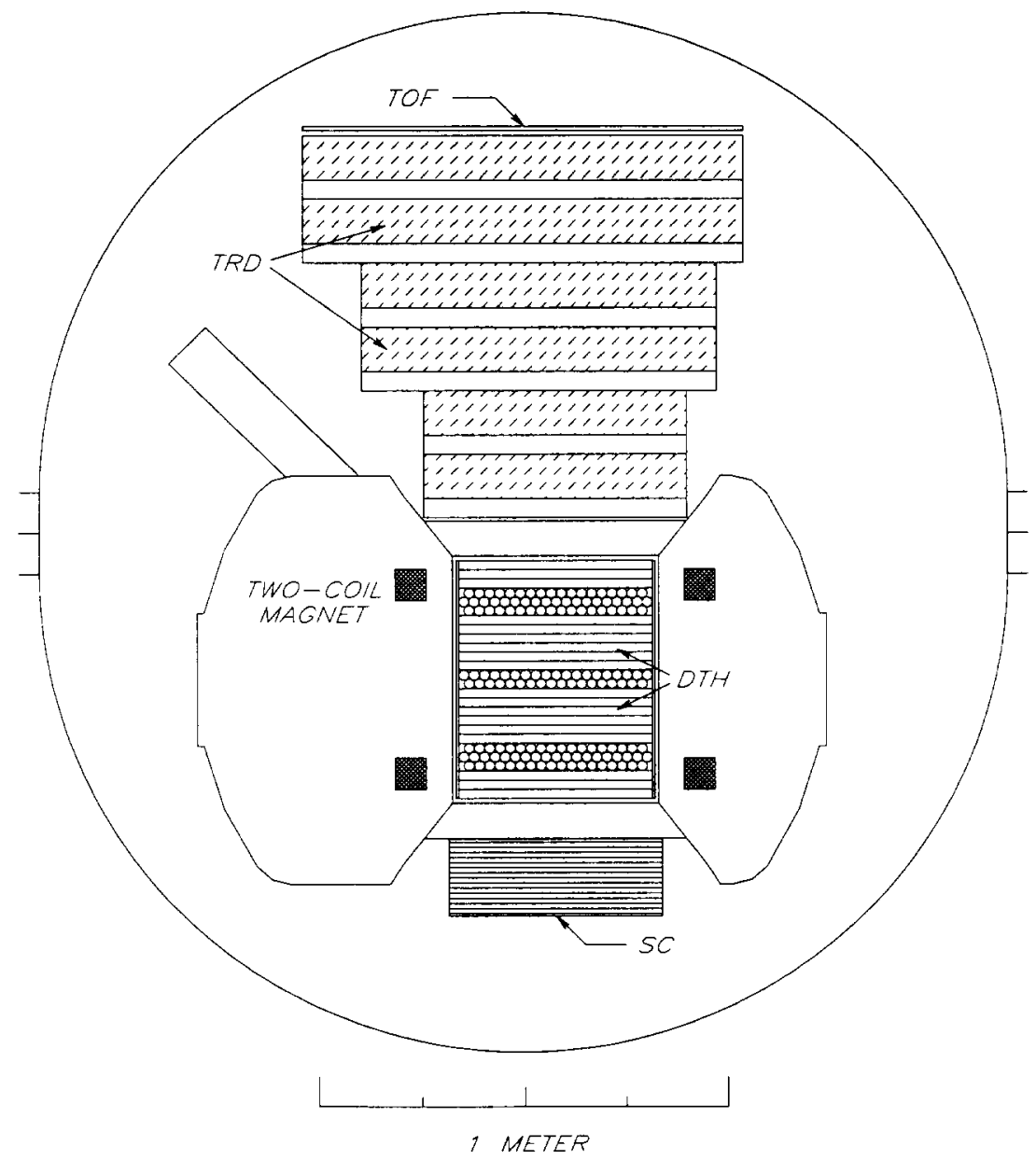

Fig. 6. The HEAT-e ${ }^{+} \mathrm{e}^{-}$spectrometer configuration. The rigidity spectrometer, consists of a drift tube hodoscope (DTH) and superconducting magnet, and is similar in design to the PBAR system. An array of six transition radiation detectors (TRD), in combination with and electromagnetic shower calorimeter (SC), suppress the proton background to the positron measurement. Upward moving particles are rejected by time-of-flight (TOF) scintillators. The top layer of SC serves as the bottom TOF counter. 


\section{Future applications}

Drift tubes have proven an ideal technology for space-based tracking hodoscopes. They provide highly redundant tracking while introducing a minimum of matter within the instrument aperture. Thin-walled tubes such as those used for SMILI and HEAT are sufficiently strong to be operated at $1 \mathrm{~atm}$ of overpressure. This eliminates the need for heavy, costly, and dangerous pressure containment vessels when designing a spectrometer to operate in the vacuum of space. When contrasted with other types of drift chambers, they are less prone to catastrophic failure, since the breakage of a single wire only renders a single tube inoperative, rather than disabling the entire tracking system. Drift gases based on $\mathrm{CO}_{2}$ and isobutane are well suited for use aboard spacecraft, since both components can be stored as liquids, eliminating the need for large, high pressure gas containers on extended missions. With the advent of long duration balloon flights from antarctica, a large aperture spectrometer such as the one used for HEAT can be used to search for low energy antiprotons with a statistically limited sensitivity of $\bar{p} / p \sim 5 \times 10^{-8}$, sufficient to detect antiprotons from relic particle annihilation in some current models.

\section{References}

[1] (The PBAR Collaboration) S.P. Ahlen, S. Barwick, J.J. Beatty, C.R. Bower, G. Gerbier, R.M. Heinz, D. Lowder, S. McKee, S. Mufson, J.A. Musser, P.B. Price, M.H.
Salamon, G. Tarlé, A. Tomasch and B. Zhou, Phys. Rev, Lett. 61 (1988) 145.

[2] (The PBAR Collaboration) M.H. Salamon et al., Ap. J. 349 (1990) 78.

[3] (The PBAR Collaboration) A.D. Tomasch et al., Nucl. Instr. and Meth. A.294 (1990) 627.

[4] A. Buffington, S.M. Schindler and C.R. Pennypacker, Astrophys. J. 248 (1981) 1179.

[5] E. Iarocci, Nucl. Instr. and Meth. 217 (1983) 30.

[6] (The SMILI Collaboration) S.P. Ahlen, J.J. Beatty, C.R. Bower, J. Clem, D.J. Ficenec, T.G. Guzik, R.M. Heinz, M. Lijowski, S. McKee, J.W. Mitchell, S.L. Mufson, J.A. Musser, S.L. Nutter, J.J. Pitts, G. Spcizak, S.M. Tobias, G. Tarlé, A.D. Tomasch, J.P. Wefel and B. Zhou, Proc. 22nd Int. Cosmic Ray Conf. 2 (1991) 563.

[7] (The SMILI Collaboration) S.P. Ahlen et al., Proc. 22nd Int. Cosmic Ray Conf. 1 (1991) 607.

[8] (The SMILI Collaboration) S.P. Ahlen et al., ibid., p. 611.

[9] (The SMILI Collaboration) S.P. Ahlen et al., ibid,, p. 615.

[10] (The HEAT Collaboration) D. Müller, S.P. Ahlen, S.W. Barwick, J.J. Beatty, A. Borione, D. Ficenec, D. Levin, D.M. Lowder, S. McKee, T. Miller, J.A. Musser, P.B. Price, S. Swordy, K.K. Tang, G. Tarlé, A.D. Tomasch and E. Torbet, Proc. 22nd Int. Cosmic Ray Conf. 2 (1991) 177.

[11] (The HEAT Collaboration) A.D. Tomasch et al., ibid., p. 181.

[12] D. Müller and K. Tang, Ap. J. 312 (1987) 183.

[13] J. Dwyer, J. Buckley, D. Müller, S.P. Swordy and K.K. Tang, Proc. 22nd Int. Cosmic Ray Conf. 2 (1991) 575.

[14] J. Buckley, J. Dwyer, D. Müller, S.P. Swordy and K.K. Tang, ibid., p. 579.

[15] J. L'Heureux, J.M. Grunsfeld, P. Meyer, D. Müller and S.P. Swordy, Nucl. Instr. and Meth. A295 (1990) 246. 